Mon. Not. R. Astron. Soc. 000,19(2017) Printed 11 September $2017 \quad$ (MN LATEX style file v2.2)

\title{
Isotropy analyses of the Planck convergence map
}

\author{
G. A. Marques ${ }^{1 \star}$ C. P. Novaes ${ }^{1} \dagger$ A. Bernui ${ }^{1} \ddagger$ and I. S. Ferreira ${ }^{2} \S$ \\ ${ }^{1}$ Observatório Nacional, Rua General José Cristino 77, São Cristóvão, 20921-400 Rio de Janeiro, RJ, Brazil \\ ${ }^{2}$ Instituto de Física, Universidade de Brasília, Campus Universitário Darcy Ribeiro, Asa Norte, 70919-970, Brasília, DF, Brazil
}

Accepted 2017 August 28. Received 2017 August 23; in original form 2017 January 04

\begin{abstract}
The presence of matter in the path of relic photons causes distortions in the angular pattern of the cosmic microwave background (CMB) temperature fluctuations, modifying their properties in a slight but measurable way. Recently, the Planck Collaboration released the estimated convergence map, an integrated measure of the large-scale matter distribution that produced the weak gravitational lensing (WL) phenomenon observed in Planck CMB data. We perform exhaustive analyses of this convergence map calculating the variance in small and large regions of the sky, but excluding the area masked due to galactic contaminations, and compare them with the features expected in the set of simulated convergence maps, also released by the Planck collaboration. Our goal is to search for sky directions or regions where the WL imprints anomalous signatures to the variance estimator revealed through a $\chi^{2}$ analyses at a statistically significant level. In the local analysis of the Planck convergence map we identified 8 patches of the sky in disagreement, in more than $2 \sigma$, with what is observed in the average of the simulations. In contrast, in the large regions analysis we found no statistically significant discrepancies, but, interestingly, the regions with the highest $\chi^{2}$ values are surrounding the ecliptic poles. Thus, our results show a good agreement with the features expected by the $\Lambda \mathrm{CDM}$ concordance model, as given by the simulations. Yet, the outliers regions found here could suggest that the data still contain residual contamination, like noise, due to over- or under-estimation of systematic effects in the simulation data set.
\end{abstract}

Key words: Cosmology - statistical isotropy - secondary anisotropies of the CMB weak gravitational lensing - Convergence map.

\section{INTRODUCTION}

Observations of the temperature and polarization of the cosmic microwave background (CMB) data provide crucial information about the early universe (see, e.g., Durrer (2008); $\mathrm{Hu}(2000))$. However, these data also encode important signatures from secondary processes, generated after the last scattering surface (LSS), which contribute to blur the primordial features of the CMB radiation (Weiland et al. 2010. Serra \& Cooray 2008; Aghanim et al. 2008). One of these secondary processes, the weak gravitational lensing (WL), consists in deflections of the CMB photons coming from the LSS by the inhomogeneous distribution of matter along our line of sight (Blanchard \& Schneider 1987; Cole \& Efstathiou 1989 Linder 1990). The main mensurable effect resulting from this phenomenon is the smoothing of the acoustic peaks

\footnotetext{
* e-mail: gabrielamarques@on.br

$\dagger$ e-mail: camilapnovaes@gmail.com

‡ e-mail: bernui@on.br

$\S$ e-mail: ivan@fis.unb.br
}

in the CMB angular power spectrum, reducing the contrast of the peaks and troughs (Lewis \& Challinor 2006). Since these data is used to assert the parameters of competing cosmological models, it is important to correctly discriminate this WL effect on CMB data.

The WL on CMB photons has been investigated and measured by various methods and diverse experiments in the recent past (Hirata et al. 2004, Smith et al. 2007; Hirata et al. 2008, Smidt et al. 2011; Das et al. 2011, Keisler et al. 2011 Feng et al. 2012b a , Van Engelen et al. 2012, Story et al. 2013: Das et al. 2014). However, only with the observations of the Planck satellite, that improved the detector sensitivity getting reliable knowledge of the noise, the dominant signal at small angular scales (Planck Collaboration et al. 2014, 2016c), it was possible the robust reconstruction of the lensing potential map, LPM, which consist in the sky projection of the gravitational potential causing the WL of the CMB photons.

The convergence map is related to the gravitational lensing potential via the 2-dimensional (2D) Poisson equation and can be interpreted as the sky projection of the sur- 
face density of the (baryonic plus dark) matter structures that CMB photons encounter in their path between the LSS and us. Because different cosmological models predict diverse scenarios of how the matter clumps, the convergence map features can be beneficial to test these possibilities, or even be used to improve parameter constraints within the concordance cosmology, $\Lambda \mathrm{CDM}$, particularly for parameters affecting the structure formation in the universe (Planck Collaboration et al. 2016a). As a matter of fact, the Cosmological Principle asserts that the matter and radiation are isotropically and homogeneously distributed around us at large scales. For this, many efforts have been done to test this property of the universe using diverse cosmological probes, like the CMB data (Ghosh et al. 2016; Schwarz et al. 2016, Bernui et al. 2014), the gamma-ray bursts (Tarnopolski 2015 Bernui et al. 2008, Ukwatta \& Woźniak|2016), the angular distribution of galaxies (Bengaly et al. 2016b Alonso et al. 2015. Tiwari \& Nusser 2016) and galaxy clusters (Bengaly et al. 2016a), for instance.

In this sense, our main motivation here is to test how isotropically distributed is the matter in the universe according to information contained in the convergence map and, moreover, if this result is in agreement with what is expected in the concordance cosmology, $\Lambda$ CDM. Subsequently, we perform analyses upon the Planck lensing data set, that is, the simulated and the estimated convergence map, evaluating how the empirical variance (following a procedure described below) is distributed in the whole sky.

This paper is organized as follows: section 2 presents a brief review about the WL effect upon the CMB photons. Details about the weak lensing products released by the Planck collaboration, besides the analyses performed upon them, are described in section 3 . The results and discussions are presented in section 4 and the conclusions are addressed in section 5

\section{WEAK GRAVITATIONAL LENSING OF THE CMB}

The deviation of the CMB photons path by potential gradients along the line of sight from the LSS until they reach us can be described by a deflection vector field, expressed as $\alpha(\hat{\Omega}) \equiv \nabla \psi(\hat{\Omega})$, where $\nabla$ is the $2 \mathrm{D}$ gradient operator on the sphere and the $\psi(\hat{\Omega})$ parameter corresponds to the lensing potential in the direction $\hat{\Omega}$. The deflection also can be related in terms of the convergence term, $\kappa=-\frac{1}{2} \nabla \cdot \alpha$.

The lensing potential $\psi$ is the projection along the lineof-sight of the gravitational potential, $\Psi(r, \hat{\Omega})$, at conformal distance $r$, that is,

$$
\psi(\hat{\Omega})=-2 \int_{0}^{r_{0}} d r \frac{d_{A}\left(r_{0}-r\right)}{d_{A}(r) d_{A}\left(r_{0}\right)} \Psi(r, \hat{\Omega}),
$$

where $r_{0}$ is the conformal distance to the LSS and $d_{A}$ is the comoving angular diameter distance. Since the convergence is related to the lensing potential via the $2 \mathrm{D}$ Poisson equation, $\kappa=-\frac{1}{2} \nabla^{2} \psi$, it can be interpreted as a (projected) surface density.

Lensing, in General Relativity, is an achromatic effect, that is, it remaps the CMB fluctuations without changing the frequency dependence and number of photons. The redistribution of the CMB temperature fluctuations on the sky through angular deflections can be expressed by $\tilde{\Theta}(\hat{\Omega})=\Theta(\hat{\Omega}+\alpha)$, where $\Theta(\hat{\Omega})=\Delta T(\hat{\Omega}) / T_{0}$, with $T_{0}=2.725 K$ (Kogut et al. 1996). The deflections of photon's paths generate distortions in the angular pattern of the primary temperature fluctuations so that, expanding the above expression in a Taylor series one can write as approximation (Lewis \& Challinor 2006)

$$
\begin{array}{r}
\tilde{\Theta}(\hat{\Omega})=\Theta(\hat{\Omega}+\nabla \psi) \simeq \Theta(\hat{\Omega})+\nabla^{a} \Theta(\hat{\Omega}) \nabla_{a} \psi(\hat{\Omega})+ \\
\frac{1}{2} \nabla^{a} \psi(\hat{\Omega}) \nabla^{b} \psi(\hat{\Omega}) \nabla_{a} \nabla_{b} \Theta(\hat{\Omega})+\ldots
\end{array}
$$

The remapping of the CMB fluctuations changes in few percent the angular power spectrum, $C_{l}^{\Theta}$, mainly at small scales $(\mathrm{Hu} 2000$, Lewis 2005). From the Taylor approximation in equation (2) the lensed power spectrum $\tilde{C}_{l}^{\Theta}$ can be written as

$$
\begin{aligned}
\tilde{C}_{l}^{\Theta} \simeq C_{l}^{\Theta} & +\int \frac{d^{2} \mathbf{l}^{\prime}}{(2 \pi)^{2}}\left[\mathbf{l}^{\prime} \cdot\left(\mathbf{l}-\mathbf{l}^{\prime}\right)\right]^{2} C_{\left|\mathbf{1}-\mathbf{l}^{\prime}\right|}^{\psi} C_{l^{\prime}}^{\Theta} \\
& -C_{l}^{\Theta} \int \frac{d^{2} \mathbf{l}^{\prime}}{(2 \pi)^{2}}\left(\mathbf{l} \cdot \mathbf{l}^{\prime}\right)^{2} C_{l^{\prime}}^{\psi}+\ldots
\end{aligned}
$$

That is, the lensed power spectrum depends on the convolution of the unlensed temperature power spectrum with the lensing potential power spectrum, $C_{l}^{\psi}$. This convolution transfers power from large to small scales making the resulting lensed power spectrum dominant over the primary one at $l \geqslant 5000$ (Hanson et al. 2010).

In addition, the effect of WL also mixes the E-mode polarization into B-mode polarization (Planck Collaboration et al. 2016e and produces a distinctive small-scale nonGaussian trispectrum (four-point correlation function), with smaller signals in the higher even n-point functions (Hanson et al. 2009). The WL effect is a secondary effect that changes slightly the primary CMB anisotropy features, however provides important cosmological constraints complementary to the CMB data (Planck Collaboration et al. 2016c) and to the large scale structure (Liu \& Hill |2015, Liu \& Haiman 2016 Liu et al. 2016, Kirk et al. 2016; Singh et al. 2017).

In the above paragraphs we summarized the expected angular distribution features of the CMB lensed photons, with no indications for preferred directions. For this, it is worth to test the statistical isotropy of the Planck WL products, at small and large regions, to investigate whether other effects (like foregrounds, masking procedure, etc.) may have introduced anomalous, and unaccounted, deviations from isotropy in the data.

\section{DATA AND METHODOLOGY}

\subsection{The Planck convergence map}

In 2015, the Planck collaboration performed a precise measurement of CMB lensing power spectrum, with a $40 \sigma$ detection (Planck Collaboration et al. 2016c). To reconstruct the CMB lensing potential map the Planck team used quadratic estimators (Okamoto \& Hu 2003) that make use of the features induced by the lensing process, that is, the diverse correlations of the CMB temperature $(\mathrm{T})$ and polarization $(\mathrm{E}$ and $\mathrm{B})$ modes. A combination of these estimators in a minimum-variance (MV) estimator was used to reconstruct the $\mathrm{CMB}$ lensing potential, $\hat{\psi}^{\mathrm{MV}}$. The $\mathrm{CMB}$ dataset used 


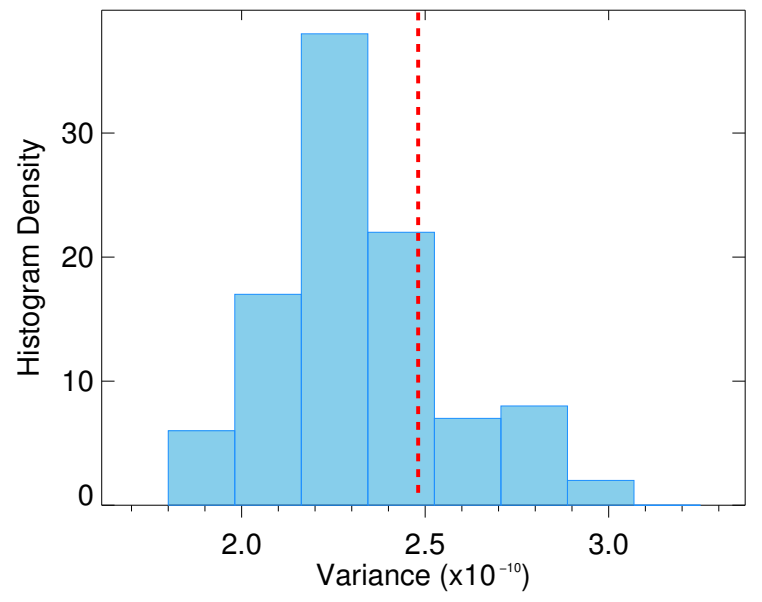

Figure 1. Histogram of the variance for the simulated Planck dataset, $\sigma_{(\text {sims })}^{2}$, calculated excluding the WL mask region. The vertical red dashed line corresponds to the variance of the Planck convergence map $\sigma_{(\text {planck })}^{2}$.

as input to the MV lensing estimator was the foregroundcleaned map obtained applying the SMICA code to the raw Planck 2015 full-mission frequency maps (Planck Collaboration et al. 2016a). The Planck team provided the multipole expansion coefficients of the estimated lensing convergence map, $\kappa$, which can be written as a function of the lensing potential spherical harmonic coefficients as

$$
\kappa_{L M}^{\mathrm{MV}}=\frac{L(L+1)}{2} \hat{\psi}_{L M}^{\mathrm{MV}}
$$

where $L$ and $M$ are the multipole components indices for the lensing reconstructed map.

The noise reduction of the maps is performed by applying a Wiener filter in spherical harmonics Bobin et al. 2012)

$$
\hat{\psi}_{L M}^{\mathrm{WF}}=\left(\frac{C_{L}^{\psi, f i d}}{C_{L}^{\psi, f i d}+N_{L}^{\psi}}\right) \hat{\psi}_{L M}^{\mathrm{MV}},
$$

where $C_{L}^{\psi, \text { fid }}$ is the lensing potential power spectrum according to the fiducial cosmological model and $N_{L}^{\psi}$ is the noise power spectrum of the $\psi$ reconstruction. The lensing convergence and the noise on $\kappa$ have a much whiter power spectrum on large scales while the estimated lensing potential has a very red power spectrum (Planck Collaboration et al. 2016a). Cutting the maps with red power spectrum in small portions can cause leakage issues. For this reason, we use in our analysis the Wiener-filtered convergence map, $\hat{\kappa}^{\mathrm{WF}}$, instead of the lensing potential map, as described in the next section. It was produced considering the resolution $N_{\text {side }}=2048$, using the HEALPix (Hierarchical Equal Area iso-Latitude Pixelization) pixelization grid (Górski et al. 2002). Our analyses are performed using the corresponding confidence mask (hereafter called WL mask), also released among the Planck lensing products. This mask joins of the Galaxy mask, that removes $f_{s k y} \simeq 0.302$, the point-sources mask that removes additional $f_{\text {sky }} \simeq 0.07$ (Planck Collaboration et al. 2014) and the SMICA specific temperature and polarization mask (Planck Collaboration et al. 2016a). The combination of these masks leaves a total unmasked sky fraction of $f_{\text {sky }} \simeq 0.673$.

\subsection{Planck Lensing simulations}

The performance of the quadratic estimators used to reconstruct the lensing maps is affected by non-lensing sources such as foreground residuals and inhomogeneous instrumental noise, besides the effects of beam asymmetry and masking. To account for these biases and to characterize the variance limits of the reconstructed convergence map $\hat{\kappa}^{\mathrm{WF}}$, we used a set of 100 realistic realizations of this observable, also released by the Planck team. These simulations are based on the Full Focal Plane 8 (FFP8) Monte Carlo realizations which is a set of maps that incorporate the dominant instrumental (detector beam, bandpass and correlated noise properties), scanning (pointing and flags) and data analysis (map- making algorithm and implementation) effects as described in Planck Collaboration et al. (2016b). The FFP8 simulations do not include galactic foregrounds contributions. In the lensing analysis, residual foregrounds in the SMICA maps are modelled by adding a small level of statistically-isotropic Gaussian noise, with an appropriate angular power spectrum, to the simulated frequency maps after combination with the SMICA weights. It is worth mentioning that the noise distribution in the data is highly anisotropic and any mis-modelling of the simulated noise may have implications for the current analysis.

All the non-lensing effects included in the reconstruction process make the $\hat{\kappa}^{\mathrm{WF}}$ data map highly non-isotropic. To study how the variance of the WL is distributed on the sky, beyond the contribution of those effects, we considered in our analysis the MV convergence simulated dataset after applying a Wiener-filter to them using equation (5), similarly to the procedure described by the Planck Collaboration to obtain the estimated convergence map.

The reconstruction of the data is band-limited to $8 \leqslant$ $L \leqslant 2048$ due the instability upon low order multipoles (Planck Collaboration et al. 2016a). For this reason, we verify that the simulated maps contain the same multipole range as well the same pixelization resolution as the Planck data.

Moreover, to confirm that the Planck simulated maps have their variances in agreement with that of the reconstructed $\hat{\kappa}^{\mathrm{WF}}$ map, we plot in figure 1 the histogram of their empirical variance upon almost full-sky, that is, cutting only the area enclosed by the WL mask. The vertical dashed line represent the variance of the estimated Planck convergence map, $\hat{\kappa}^{\mathrm{WF}}$. The average value from the 100 simulated maps is in complete agreement with the variance of the data map, that is, they have the same value up to $1 \sigma$.

\subsection{Analyses of the Planck convergence map}

The main goal of our analyses is to test how isotropic is the Planck convergence map. The variance provides a simple way to look for statistically significant deviations compared to the expected by the simulated convergence maps in different regions of the sky.

In this sense, we choose to perform our analysis of the convergence maps using two complementary approaches: (1) 


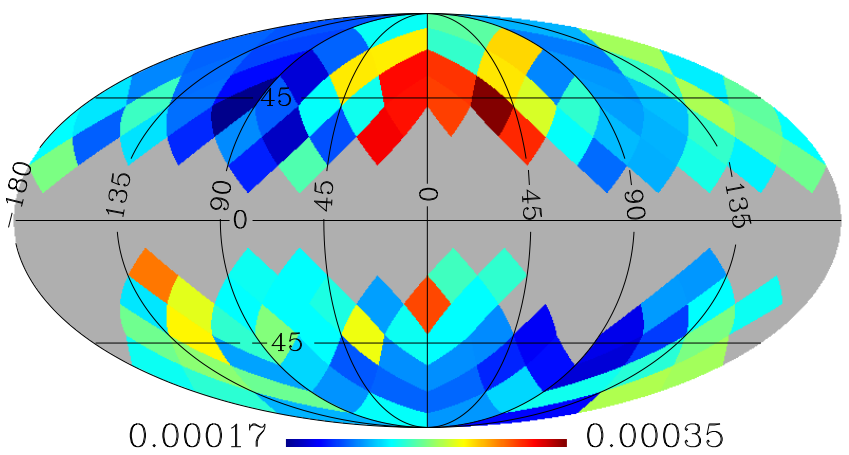

Figure 2. Mollweide projection of the variance $\sigma_{p(\text { Planck })}^{2}$ calculated in patches of the estimated Planck convergence map, $\hat{\kappa}^{W F}$ (see text for details). The region excluded from the analysis by the WL mask is presented in grey.

performing local analyses calculating the lensing variance in non-overlapping patches of the sky, and (2) through a hemispherical scan of the sky, in order to determine if there is a preferred direction, statistically significant in comparison to the expected from the simulations. Each method is described in detail below.

\subsubsection{Local analysis}

We define the sky regions where to carry out the local analysis as being the pixels corresponding to a HEALPix resolution of $N_{\text {side }}=4$, that is, 192 pixels of equal area $\sim(14.7 \mathrm{deg})^{2}$. Each one of these big pixels in the small resolution (hereafter called patches) contains $\sim 262,144$ small pixels in the high resolution, $N_{\text {side }}=2048$.

Since we used the WL mask to remove Galactic and extragalactic contamination, the number of valid pixels in each region, after the application of the mask, varies from one region to another. Then, we established the criterion of discarding the patches whose number of valid pixels is less than $80 \%$ of the total. As a result, a total of 116 patches were selected for analysis.

Aware of how the WL effects are distributed on the sky, our analysis uses the variance of the lensing convergence as the statistical estimator to measure the directional amplitude of this effect. For each of the $p$-patches $(p \in[1,116])$, we calculate this quantity in all the simulated, $\sigma_{p(\text { Sims })}^{2}$, and Planck estimated, $\sigma_{p(\text { Planck })}^{2}$, convergence maps.

The figure 2 shows, in color scale, a Mollweide projection of the $\sigma_{p \text { (Planck) }}^{2}$ for each of those patches, and the figure 3 presents the mean variance, $\left\langle\sigma_{p(\text { Sims })}^{2}\right\rangle$, calculated from the simulated dataset.

\subsubsection{Hemispherical analysis}

In contrast, the hemispherical analysis applies the empirical variance estimator upon large regions of the sky. The centres of the hemispheres are defined by pixelizing the celestial sphere according to the resolution parameter $N_{\text {side }}=4$. This provides a total of 192 coordinates centres. Once more, we used the WL mask to remove the residual contamination region. Since in the current case we are considering regions

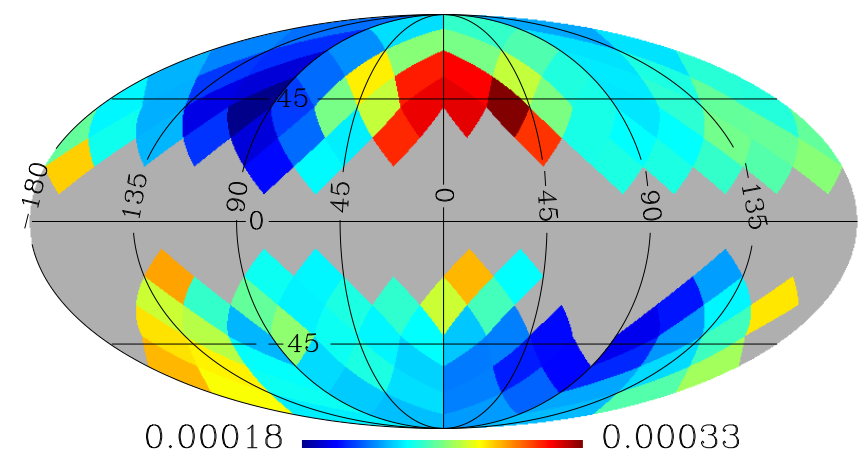

Figure 3. Mollweide projection of the mean variance $\left\langle\sigma_{p(\text { Sims })}^{2}\right\rangle$, similar to the analysis presented in figure 2 but averaging upon the 100 simulated convergence maps (see text for details).

corresponding to hemispheres, which encompasses large areas of the sky, the percentage of valid pixels variate slightly in each of them.

Similarly to the local analysis, we calculate the variance in each one of the $h$-hemispheres, $h \in[1,192]$, upon all the simulated, $\sigma_{h(\text { Sims })}^{2}$, and estimated, $\sigma_{h(\text { Planck })}^{2}$, Wienerfiltered convergence maps. The Mollweide projection of the hemispherical variance of the reconstructed Planck convergence map, $\sigma_{h(\text { Planck })}^{2}$, and the corresponding average from the simulated dataset, $\left\langle\sigma_{h(\operatorname{Sims})}^{2}\right\rangle$, are shown in figures 4 and 5 respectively.

\section{RESULTS AND DISCUSSION}

We analyzed the statistical isotropy of the simulated and estimated Planck convergence maps using the variance as statistical estimator calculated in regions defined according to two approaches, small and large regions, as discussed in section 3 As a result we observe that the variance from the data map is not isotropically distributed in the sky in none of the cases. Moreover, the comparison between the angular distribution of the variance on sky for the estimated convergence map and the average from simulated maps showed up a strong correlation in both, the local (figures 2 and 3 ) and hemispherical (figures 4 and 5 ) analyses, as shown in the correlation plots in figure 6

Such correlation would be an indication that the contributions coming from diverse non-lensing processes may have introduced the anisotropies imprinted in the variance distribution. An example is the Planck scanning strategy, which makes the instrumental noise to be minimised close to the ecliptic poles. Accordingly, the minimum variance values obtained from the local analysis of the convergence data, as well as those from the simulations (figures 2 and 3), are displayed around the ecliptic poles. This corroborates the efficiency of the variance estimator, upon small and large regions, not only to probe the lensing signal, but also the residual contamination in the data and accounted in the simulations.

Even visually correlated, observing the color scales of the Mollweide projections in figures 2 and 3 the variance in the estimated Planck map still appears to have some regions with higher/lower amplitudes compared to the average from 


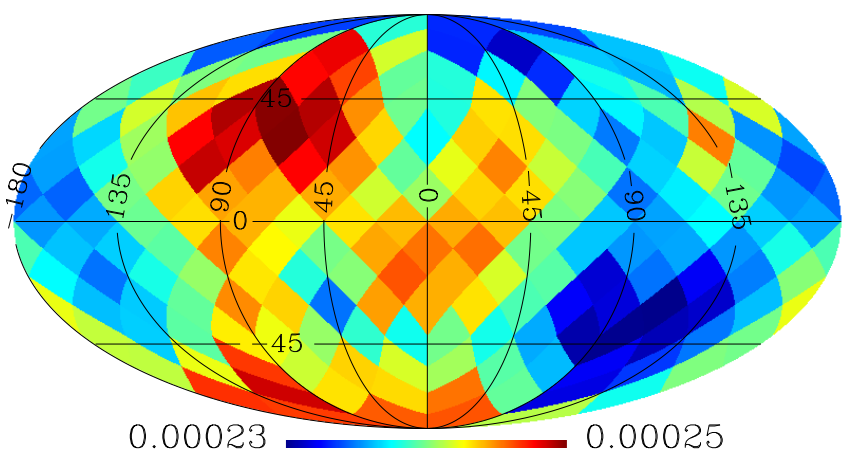

Figure 4. Mollweide projection of the variance, $\sigma_{h(\text { Planck })}^{2}$, obtained performing the hemispherical analysis of the Planck convergence map, $\hat{\kappa}^{W F}$, and projected at the 192 centres, as explained in the text.

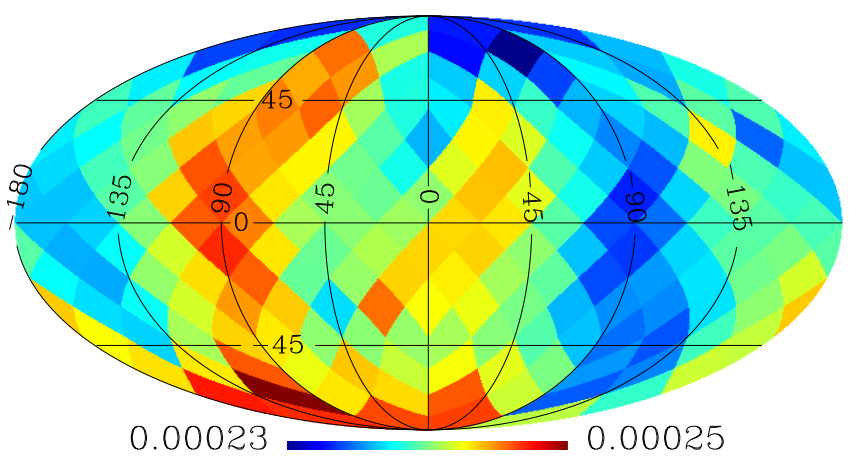

Figure 5. Mollweide projection of the $\left\langle\sigma_{h(\text { Sims })}^{2}\right\rangle$ resulting from a hemispherical analysis, similar to the analysis presented in figure 4 but averaging upon the values obtained from the 100 Planck simulated convergence maps (see text for details).

the simulated maps. In order to evaluate the possibility of having regions of the $\hat{\kappa}^{W F}$ map with anomalously high (or low) variance values, we establish a comparison between its corresponding variance map and the one obtained from the simulations through a $\chi^{2}$ analysis. Then, for each of the $i=h$ th hemisphere, with $h=1, \ldots, 192$, and $i=p$ th patch, $p=1 \ldots, 116$, we perform the calculation of

$$
\chi_{i}^{2}=\left(\frac{\sigma_{i(\text { Planck })}^{2}-\left\langle\sigma_{i(\text { Sims })}^{2}\right\rangle}{\Sigma_{i}}\right)^{2},
$$

where $\left\langle\sigma_{i \text { (Sims) }}^{2}\right\rangle$ and $\Sigma_{i}$ are the average and standard deviation over the variance sample of 100 simulated maps.

Associating to each region its corresponding $\chi^{2}$ value lead us to build a $\chi_{p}^{2}$-map for the local analysis and a $\chi_{h^{-}}^{2}$ map for the hemispherical one. These maps provide valuable informations about the contribution of the WL effect under different local regions and directions of the sky.

The variance of the $\hat{\kappa}^{W F}$ and the mean variance of the simulated maps are in agreement on less than $1 \sigma$ in the whole sky analysis- excluding only the portion of the WL mask as shown in the figure 1 .

In fact, according to the $\chi_{p}^{2}$ distribution from the local analysis, showed in figure 7 most of the patches have a $\chi_{p}^{2}$ value very close to zero, indicating this agreement in
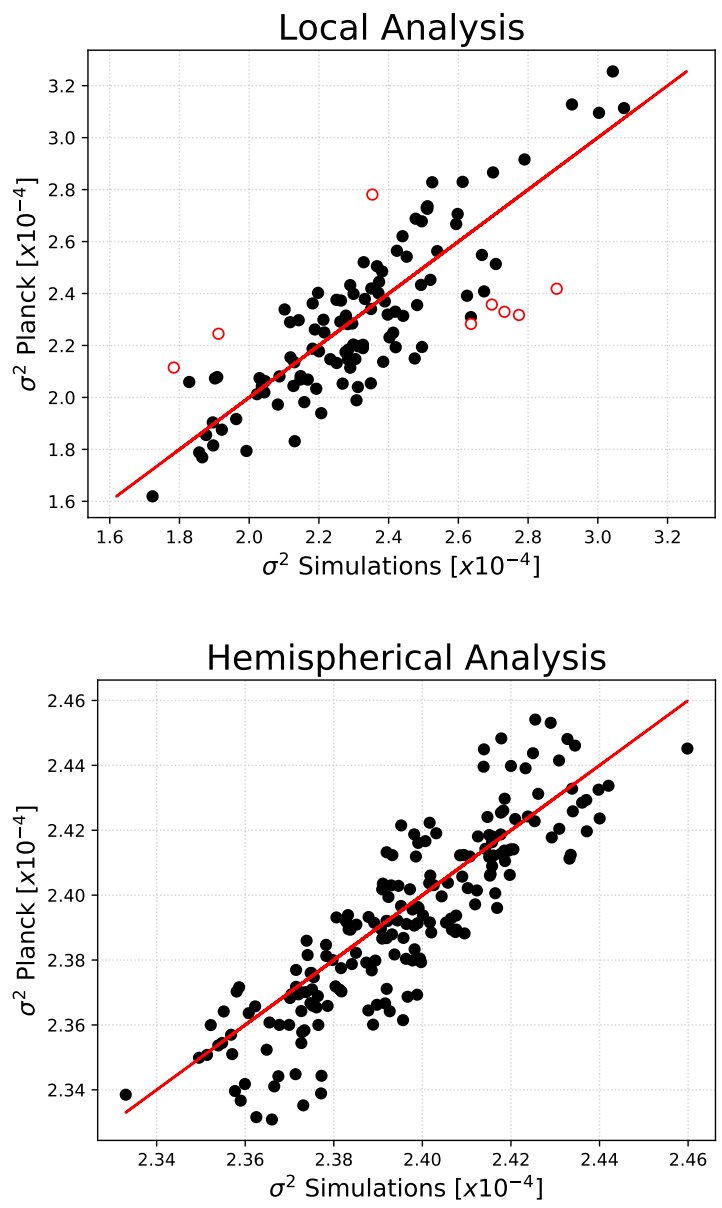

Figure 6. Correlation among the average variance from simulations (horizontal axis) and the variance from estimated (vertical axis) convergence maps for local and hemispherical analyses. The red open circles in the local analysis plot correspond to the regions which the estimated Planck convergence map deviate from the mean of the simulations more than $2 \Sigma$, as identified by the $\chi^{2}$ analysis (see text for details)

those regions. For this reason, only the upper bounds of $68 \%$ (yellow dashed line) and 95\% (red dashed line) Confidence Levels (CL) are shown in figure 7. However, we still find 8 patches whose $\chi_{p}^{2}$ values indicate that the variance of the data disagree in more than $2 \Sigma$ (i.e., $95 \% \mathrm{CL}$ ) from the mean value of the simulations.

These 8 patches are represented as red open circles in the analysis shown in the upper panel of figure 6. Note that the farther the symbols from the red diagonal line, the equality line $\left(\sigma_{p(\text { Planck })}^{2}=\left\langle\sigma_{p(\text { Sims })}^{2}\right\rangle\right)$, higher the disagreement among the variance values from estimated and simulated convergence maps. The analysis presented in figure 7 show how discrepant are the $\chi_{p}^{2}$ values of these 8 patches relatively to the others. In terms of the $\Sigma$ deviations of the variance from the mean of the simulations, the statistical significances of these patches, namely, $p=68,10,80,8,42,97,114^{1}$ and

\footnotetext{
1 Notice that the numeration of the patches centres does not corresponds to the numeration of the hemispheres centres because 76 patches were excluded by the WL mask.
} 


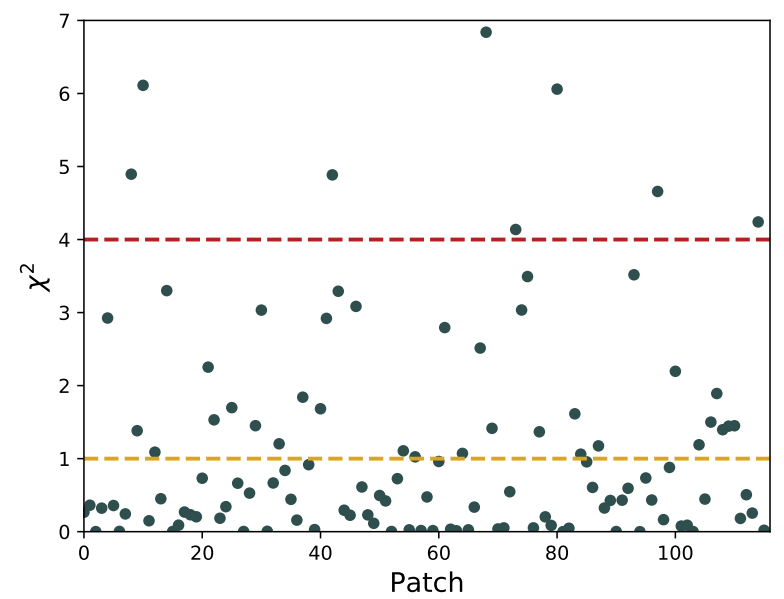

Figure 7. $\chi_{p}^{2}$ values as a function of the patch number $p$, with $p=$ $1, \ldots, 116$. The dashed lines represent the corresponding $1 \Sigma(68 \%$ CL; green) and $2 \Sigma(95 \% \mathrm{CL}$; red) confidence regions, respectively.

73 , are $2.61,2.47,2.46,2.21,2.21,2.15,2.05$, and $2.03 \Sigma$, respectively. Although do not seem to exist a statistically expressive discrepancy among data and simulations, these analyses identify outliers regions that may be associated to features of the lensing signal, or even some deficiency in the simulations. The $\chi_{h}^{2}$ values for hemispherical analysis as a function of the hemisphere number are shown in figure 8 . The 1 and $2 \Sigma$ levels in this case are also represented in the figure as yellow and red dashed lines respectively. Similarly to the local analysis, most of the hemispheres of the estimated convergence map have their variance very close to those obtained from the simulations, so the corresponding $\chi_{h}^{2}$ values are also quite small. In contrast to the local analysis results, the variance of the data upon all the hemispheres seems to be in agreement with the average from the simulations in less than $2 \Sigma$. For this reason, there is no red open circles in the bottom panel of figure 6 as in the local results. However, it is worth mentioning that the hemispheres with the greatest deviations have their variance values $1.84,1.71,1.68$ and $1.66 \Sigma$, whose coordinates centres are $h=146,42,164$ and 129 , respectively.

The figure 9 shows, in color scale and Galactic coordinates, the sky projection of the outliers patches identified in the $\chi_{p}^{2}$-map. They do not appear to be concentrated in a particular region of the sky. However, one observes that some of these patches are located near the region defined by the WL mask, suggesting that residual foregrounds could still be affecting the analyses.

Due to the different sizes encompassed in the local and hemispherical analyses, they probe different but complementary aspects. The hemispheres are composed by regions of $90^{\circ}$ radius, probing effects dominant in large areas of the sky, while local analysis might reveal the effect of small structures or localized residual foregrounds. Unlike the local analysis, our results did not reveal outliers hemispheres, that is, where deviations are above $2 \Sigma$ level (95\% CL). For this, we show in the figure 10 the all sky projection of the $\chi_{h}^{2}$-map.

Although there are no significant deviations resulting from the hemispherical analysis, curiously, the regions with highest $\chi^{2}$, that is, the larger discrepancy among data and

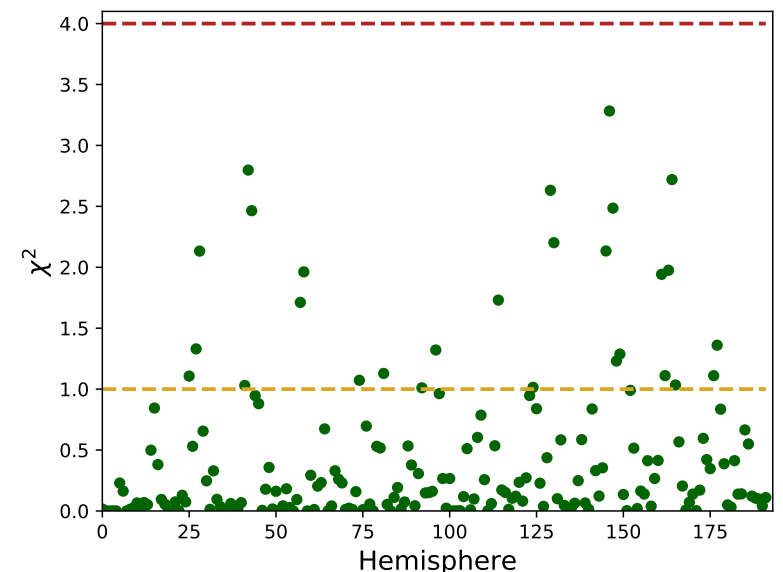

Figure 8. $\chi_{h}^{2}$ values as a function of the hemisphere number $h$, with $h=1, \ldots, 192$. The dashed lines represent the $1 \Sigma(68 \% \mathrm{CL}$; yellow) and $2 \Sigma(95 \%$ CL; red) confidence regions, respectively.

simulations, are concentrated around the ecliptic poles. Note that the hemispheres centred near the ecliptic poles are those where noise modelling could be the most problematic. This is expected because these hemispheres encompass regions where the noise levels are higher than average (i.e., near the galactic plane) and where deviations from statistical isotropy are expected due to the Planck scanning strategy.

The Planck convergence MV map used in our isotropy analyses is estimated from an optimized reconstruction using a quadratic estimator which measures the lensing induced in a CMB map, specifically, in the foreground-cleaned SMICA map (Planck Collaboration et al. 2016a). However, as discussed by, e.g., Planck Collaboration et al. (2016d); Novaes et al. (2016); Schwarz et al. (2016), the procedures used to obtain the four foreground-cleaned Planck CMB maps, among them the SMICA, even though highly efficient, could have leaved undesired residuals and some artifacts. Although the set of simulated maps take into account these effects, they might be under- or over-estimated. For example, the lack of accuracy of the statistically-isotropic and Gaussian field added to the simulations to take into account the noise in the data. In this sense, other sources of statistical anisotropy can also be the responsible for the small deviations of the variance observed in our analyses.

Moreover, it is important to be sure that our results are not being influenced by the cut-sky mask applied to the convergence maps. As discussed before, all our analyses are performed considering a sky cut given by the WL mask, which means that each sky patch selected for the local analyses are composed by a different number of valid pixels, as well as in the case of the hemispherical analysis. In this case, to look for a possible influence of the mask in the local analysis, we analyse in figure 11 the dependence between the $\chi_{p}^{2}$ values and the percentage of valid pixels for each of the 116 patches. In this figure one observes that do not seem to exist a correlation between these two quantities. In the case of the hemispherical analyses, the dependence of the $\chi_{h}^{2}$ with the number of valid pixels are shown in the figure 12 Also, does not appear a clear dependency between these two quantities, confirming that all the information provided by the $\chi_{p}^{2}$-map and $\chi_{h}^{2}$-map would come essentially from the $\hat{\kappa}^{\mathrm{WF}}$. 


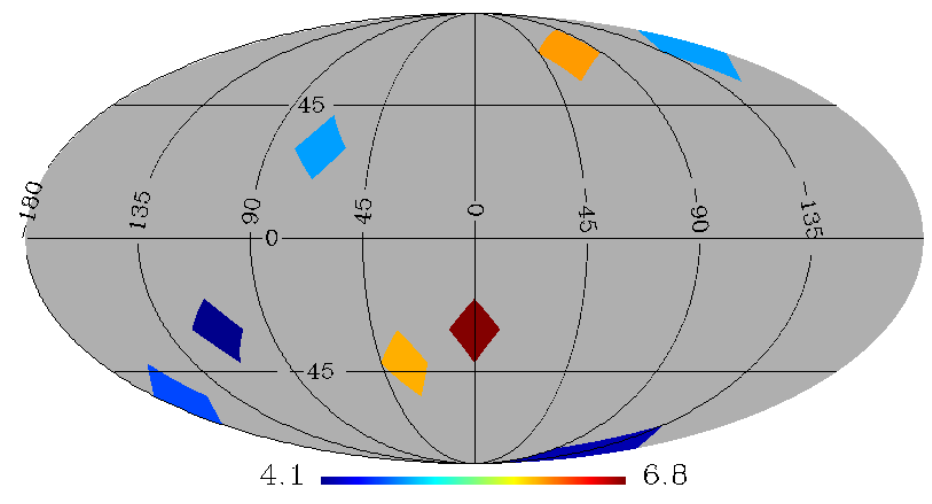

Figure 9. Mollweide projection, in color scale, of the $\chi_{p}^{2}$-map, highlighting only the outliers patches, i.e., those whose $\chi_{p}^{2}$ values are out of the $2 \Sigma$ confidence level (see figure 7 ).

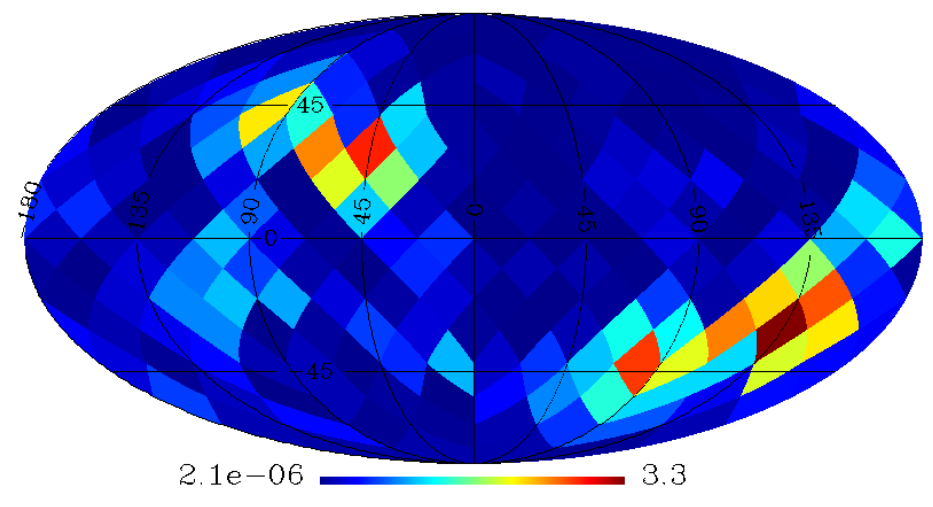

Figure 10. Mollweide projection, in color scale, of the $\chi_{h}^{2}$-map, for all of the 192 hemispheres centres (see figure 8).

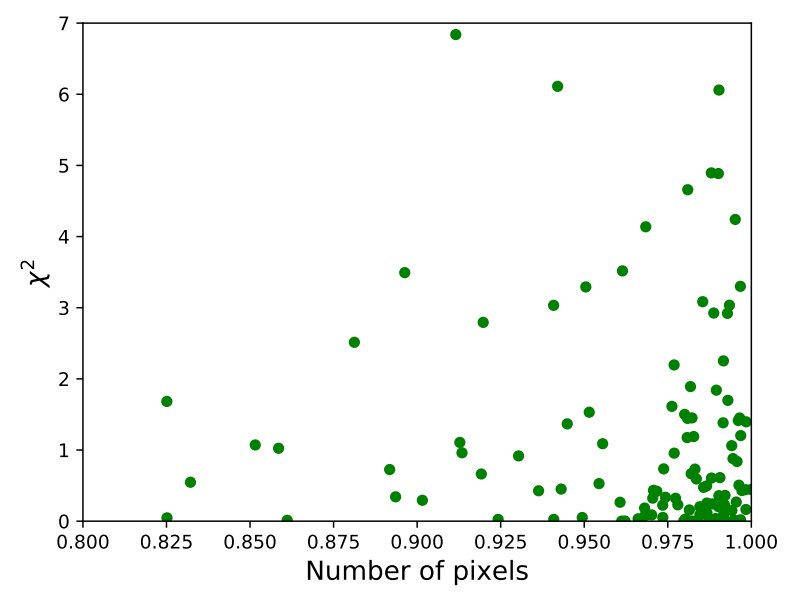

Figure 11. Dependence of the $\chi_{p}^{2}$ values with the percentage of valid pixels in the 116 patches. Notice that we are considering for analyses only the patches with a minimum of $80 \%$ of valid pixels.

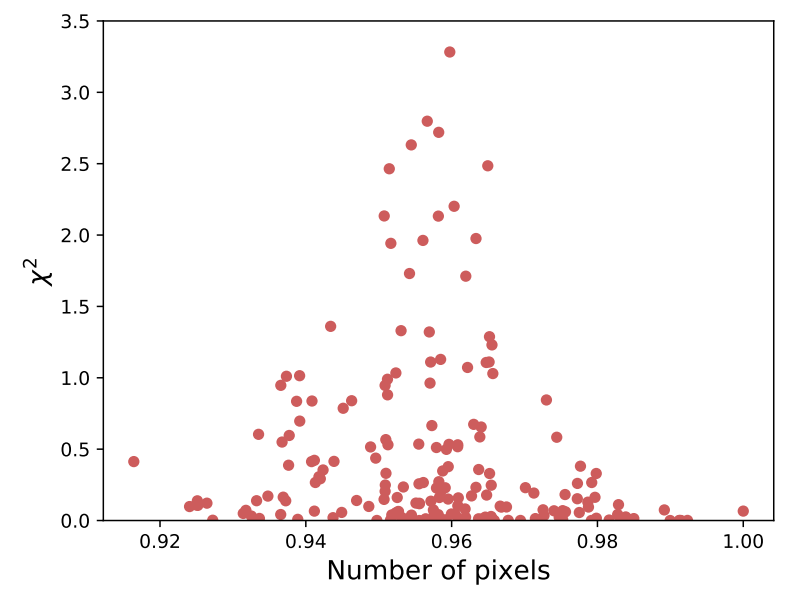

Figure 12. Dependence of the $\chi_{h}^{2}$ values with the percentage of valid pixels in the 192 hemispheres.

\section{CONCLUSIONS AND FINAL REMARKS}

The WL is a secondary effect and despite its low amplitude, it modifies some features of the primary anisotropies of the CMB. The convergence map represents an integrated measurement of the total matter distribution in the observable universe, since it is directly related to the mass inhomogeneities along the line of sight (Lewis \& Challinor 2006. Planck Collaboration et al. 2016c). Accordingly, the study of the CMB lensing phenomenon can provide direct information about the large-scale distribution of matter and, by extension, about how isotropically it is distributed in the universe.

We have presented here statistical isotropy analyses of the CMB lensing phenomenon. In order to check if there are regions of the sky exceptionally influenced by this effect we use the amplitude of the variance of the convergence map estimated by the Planck team. To do this, we analysed the sky data comparing the variance value in the estimated Planck convergence map, $\hat{\kappa}^{M V}$, with the simulated set of convergence maps. We performed two types of complementary analyses, upon small and large regions, each one considering a different criterion to select the regions where to apply our estimator.

In these analyses we calculated the variance in small patches and in hemispherical regions of the sky in two datasets, the estimated Wiener-filtered convergence map, $\hat{\kappa}^{W F}$, and in 100 simulated convergence Wiener-filtered maps, that mimic the same effects and properties of the $\hat{\kappa}^{W F}$ map (Planck Collaboration et al. 2016c b). Through a $\chi^{2}$ analyses our two approaches identify some regions of the estimated convergence map with variance larger than expected as compared with simulations. The obtained results lead us to our main conclusions:

- The local analyses highlighted 8 of the 116 regions. They correspond to regions where the variance reveals to be higher, or smaller, than the expected from the same sky regions of the simulated maps, disagreeing in more than $2 \Sigma$ (95\% CL). The distribution of these patches are spread in different parts of the sky, but two of them are near the Galactic WL mask, suggesting a possible contamination by foreground residuals. In other words, although do not exist a 
highly significant tension among the variance of the data and the mean from simulations, our analysis identified outliers regions that may be associated to some high/low lensing contribution.

- The $\chi_{h}^{2}$-map obtained from the hemispherical analysis reveals that when evaluating large regions of the sky, the data seem to be in better agreement with the simulations, since no outlier region (i.e., discrepancy of the variance values in more than $2 \Sigma$ ) was identified in this case. This indicates that the effects responsible for the outlier patches are in fact of local origin, having their signal diluted when analysing large regions. However, even though not statistically significant, it is worth to point out that the locations of the coordinate centres of the hemispheres with the highest $\chi_{h}^{2}$ values are coincidentally concentrated close to the north and south ecliptic poles.

The convergence map reveals a sum of the lens effect from near to distant structures. Generically, our results may be indicating a possible residual contamination in the CMB data, or even a true imprint left by anomalous density regions in the convergence map. In case it comes from the matter distribution of the large scale structure, further investigation of the signal, not only of its source but also the scale it appears, is crucial to evaluate the validity of the cosmological principle. However, it is also possible that our findings are related to the presence of unaccounted contributions of Galactic and extragalactic signals in the Planck CMB data, or even coming from the reconstruction process of the convergence map, as previously discussed. Aware of this, the distribution observed in the $\chi_{p}^{2}$-map indicates either the presence of some local effect in the estimated Planck convergence map or some over- or under-estimated residual effect in the simulated dataset. Moreover, these effects are diluted when performing the hemispherical analysis, results that suggest the presence of a residual anisotropic contamination, which could be originated by a mis-modelling of the additional noise included in the simulations.

In this scenario, it is clear the importance of performing the types of complementary analyses shown here. First, as a test of the statistical isotropy of the universe using the CMB lensing data, a very powerful probe of the large-scale structure at high redshifts. In fact, according to our results this observable seems to be in good agreement with what is expected in the concordance $\Lambda \mathrm{CDM}$ model. Second, to explore how the noise is distributed in the lensing data to be taken into account in future simulations. Our estimator complements other analyses helping to identify possible additional signals, that can be corroborated in future analyses with deep large-scale structure tracers. This comparison between data and simulations at small and large regions are a complementary and independent tests of the procedures used by the Planck collaboration in their attempt to simulated the data, accounting for the noise, foregrounds, and systematics of the measurements.

In fact, several works use the estimated Planck convergence map in some small specific region of the sky in order to obtain cosmological information along with large-scale structure data (Kirk et al. 2016, Singh et al. 2017, Liu \& Hill 2015, Hand et al. 2015). This turns clear the importance of confirming whether the regions identified in the present work can offer some extra cosmological information.
Finally, as a matter of fact, our analyses have shown that even though the universe seems to be in agreement with the principle of statistical isotropy, with no apparent preferred direction, we still observe localized regions that deserve future detailed analyses. The scrutiny of these regions can lead us not only to find a possible correlation with under- or over-densities in the universe, but also to a better comprehension of how additional sources (besides those considered in the set of simulated Planck's convergence maps) could be affecting cosmological analyses.

\section{ACKNOWLEDGEMENTS}

The authors thank CAPES, CNPq for the financial support. We acknowledge a CAPES PVE project (88881.064966/2014-01) within the Science without Borders Program. CPN is also supported by the DTI-PCI Programme of the Brazilian Ministry of Science, Technology and Innovation (MCTI). ISF thanks CNPqs grant PDE(234529/2014-08), and also FAPDF. The authors also acknowledge the HEALpix package for the derivation of the analyses performed in this work. We would like to thank the anonymous referee for very useful comments and feedback on this paper.

\section{REFERENCES}

Aghanim N., Majumdar S., Silk J., 2008, Reports on Progress in Physics, 71, 066902

Alonso D., Salvador A. I., Sánchez F. J., Bilicki M., GarcíaBellido J., Sánchez E., 2015, Monthly Notices of the Royal Astronomical Society, 449, 670

Bengaly C. A. P., Bernui A., Ferreira I. S., Alcaniz J. S., 2016a, Monthly Notices of the Royal Astronomical Society Bengaly C., Bernui A., Alcaniz J., Xavier H., Novaes C., 2016b, Monthly Notices of the Royal Astronomical Society, 464, 768

Bernui A., Ferreira I., Wuensche C., 2008, The Astrophysical Journal, 673, 968

Bernui A., Oliveira A., Pereira T., 2014, Journal of Cosmology and Astroparticle Physics, 2014, 041

Blanchard A., Schneider J., 1987, Astronomy and Astrophysics, 184, 1

Bobin J., Starck J.-L., Sureau F., Fadili J., 2012, Advances in Astronomy, 2012

Cole S., Efstathiou G., 1989, Monthly Notices of the Royal Astronomical Society, 239, 195

Das S., et al., 2011, Physical Review Letters, 107, 021301

Das S., et al., 2014, Journal of Cosmology and Astroparticle Physics, 2014, 014

Durrer R., 2008, The cosmic microwave background. Vol. 401, Cambridge University Press Cambridge

Feng C., Keating B., Paar H. P., Zahn O., 2012a, Physical Review D, 85, 043513

Feng C., Aslanyan G., Manohar A. V., Keating B., Paar H. P., Zahn O., 2012b, Physical Review D, 86, 063519

Ghosh S., Kothari R., Jain P., Rath P. K., 2016, Journal of Cosmology and Astroparticle Physics, 2016, 046 
Górski K. M., Banday A., Hivon E., Wandelt B., 2002, in Astronomical Data Analysis Software and Systems XI. p. 107

Hand N., et al., 2015, Physical Review D, 91, 062001

Hanson D., Smith K. M., Challinor A., Liguori M., 2009, Physical Review D, 80, 083004

Hanson D., Challinor A., Lewis A., 2010, General Relativity and Gravitation, 42, 2197

Hirata C. M., Padmanabhan N., Seljak U., Schlegel D., Brinkmann J., 2004, Physical Review D, 70, 103501

Hirata C. M., Ho S., Padmanabhan N., Seljak U., Bahcall

N. A., 2008, Physical Review D, 78, 043520

Hu W., 2000, Physical Review D, 62, 043007

Keisler R., et al., 2011, The Astrophysical Journal, 743, 28

Kirk D., et al., 2016, Monthly Notices of the Royal Astronomical Society, 459, 21

Kogut A., Banday A., Bennett C., Górski K., Hinshaw G., Smoot G., Wright E., 1996, The Astrophysical Journal Letters, 464, L5

Lewis A., 2005, Physical Review D, 71, 083008

Lewis A., Challinor A., 2006, Physics Reports, 429, 1

Linder E. V., 1990, Monthly Notices of the Royal Astronomical Society, 243, 353

Liu J., Haiman Z., 2016, Physical Review D, 94, 043533

Liu J., Hill J. C., 2015, Physical Review D, 92, 063517

Liu J., Hill J. C., Sherwin B. D., Petri A., Böhm V., Haiman Z., 2016, Physical Review D, 94, 103501

Novaes C., Bernui A., Marques G., Ferreira I., 2016, Monthly Notices of the Royal Astronomical Society, 461, 1363

Okamoto T., Hu W., 2003, Physical Review D, 67, 083002

Planck Collaboration et al., 2014, A\&A, 571, A17

Planck Collaboration et al., 2016a, A\&A, 594, A9

Planck Collaboration et al., 2016b, Astronomy \& Astrophysics, 594, A12

Planck Collaboration et al., 2016c, A\&A, 594, A15

Planck Collaboration et al., 2016d, A\&A, 594, A16

Planck Collaboration et al., 2016e, Astronomy \& Astrophysics, 596, A102

Schwarz D. J., Copi C. J., Huterer D., Starkman G. D., 2016, Class.Quant.Grav. 33, no. 18, 184001

Serra P., Cooray A., 2008, Physical Review D, 77, 107305

Singh S., Mandelbaum R., Brownstein J. R., 2017, Monthly

Notices of the Royal Astronomical Society, 464, 2120

Smidt J., Cooray A., Amblard A., Joudaki S., Munshi D., Santos M. G., Serra P., 2011, The Astrophysical Journal Letters, 728, L1

Smith K. M., Zahn O., Dore O., 2007, Physical Review D, 76,043510

Story K., et al., 2013, The Astrophysical Journal, 779, 86

Tarnopolski M., 2015, arXiv preprint arXiv:1512.02865

Tiwari P., Nusser A., 2016, Journal of Cosmology and Astroparticle Physics, 2016, 062

Ukwatta T., Woźniak P., 2016, Monthly Notices of the Royal Astronomical Society, 455, 703

Van Engelen A., et al., 2012, The Astrophysical Journal, 756,142

Weiland J., et al., 2010, arXiv preprint arXiv:1001.4731 